\title{
Potential Solutions to Selected Consequences of the COVID-19 Pandemic on Mental Health Relevant Also for the Post-COVID Era
}

\section{J. Masan (Jan Masan)¹, M. Hamarova (Mariana Hamarova)²}

${ }^{1}$ University of Thessaly, GR.

${ }^{1}$ University of Ss. Cyril and Methodius in Trnava, SK.

${ }^{1,2}$ St. Elizabeth University of Health and Social Sciences, Bratislava, SK.

\section{E-mail address:}

masanjan@gmail.com

\section{Reprint address:}

Jan Masan

Nalepkova 1

92101 Piestany

Slovakia

Source: Clinical Social Work and Health Intervention

Pages: $9-14$

Volume: 12

Issue: 3

\section{Reviewers:}

Mageswaari Rajoo

Bangkok, Thailand

Michael Costello

University of Scranton School of Education, USA

\section{Keywords:}

COVID-19. Psychological Consequences of COVID-19. Intervention Strategies.

Virtual Reality. Post-Covid Era.

\section{Publisher:}

International Society of Applied Preventive Medicine i-gap

CSWHI 2021; 12(3): 9 - 14; DOI: 10.22359/cswhi_12_3_01 C Clinical Social Work and Health Intervention

\section{Abstract:}

This article is based on the results of a survey conducted during the period from December 2020 to May 2021 involving 300 respondents in different countries: Italy, Spain, Hungary, Great Britain, the USA, Poland, Slovakia, Romania, Serbia, Ukraine, and African countries. The research was carried out on the basis of narrative case studies. In order to obtain an adequate methodological answer in regard to the focus of the research, which showed the personal and subjective views of the respondents, a qualitative approach using in-depth online interviews was desirable. Each interview lasted 90 minutes. The aim of the survey was to find out how designing your own online intervention program can help respondents alle- 
viate their difficulties. It is based on human-centered approaches; methods of reducing stress; methods of strengthening the presence-focused mode; the so-called 'here and now' approach; and some relevant theses of Christian missiology and non-radicalized Islam that promote human health and dignity. The condition for entry into the survey was the age of the participants (over 40) and experience of at least one of the psychological consequences of, or situations in, a long-term psychological burden due to the COVID-19 pandemic. Survey participants became infected with COVID-19 or had recovered from it before entering the survey. Many of them had other associated health problems, including COVID-19 and its psychological consequences, chronic illnesses, were at risk of poverty, social exclusion or insufficient professional health and social care. During the survey, the online intervention program focused on gradually accompanying survey participants towards their full autonomy (for example, in thinking, naming the feelings they experience), encouraging them to take responsibility for their lives and encouraging them by positively evaluating their achievements and the results they achieved over the course of the year. The participants in the survey showed a significant weakening of rumination, which is behind the psychological difficulties, especially mood disorders and anxiety disorders. The online intervention program worked intensively with survey participants in line with the need to activate their change process. The intention of the online intervention program was also to help them regain their inner balance and their full place in society they had before the pandemic. The survey showed that even in the post-COVID period, special online strategies aimed at promoting mental and physical health should not lose their relevance. The results of such research may provide relevant and inspiring stimuli for further and new specific research, studies and analyses in the field of the benefits of specialized online interventions aimed at strengthening physical and mental health. Especially during heightened critical periods, which global crises and their consequences bring into various areas of life for the entire population. These are, for example, crises in the form of other pandemics, such as the COVID-19 pandemic, which has had a far-reaching impact on today's society.

\section{Introduction}

Experience and recent knowledge gained during the COVID-19 pandemic have shown that some of the serious and wide-ranging negative effects of the pandemic include, in particular, those that affect mental health and resilience. Billions of people all over the world experienced anxiety; unease; stress; worries; social isolation; loneliness; a temporary state of depression; feel- ings of loss of control over the situation and over their emotions; various consequences of their worsening economic situation; the negative effects of isolation on their partnerships, intimacy and family life; anticipated anxiety caused by fake news related to the pandemic which often came from unauthoritative sources. Similar findings were presented in research carried out by Jiju Varghese et al. in 2020 (1), which focused 
on negative consequences related to the COVID19 pandemic, for instance: "anxiety and fear of danger; intense feelings of losing control over one's life; negative emotions; depression; disorientation; psychosomatic disorders; aggression; increased risk of being manipulated or controlled; reduced ability to distinguish and analyze information" (1). Long-lasting tense mental and emotional experiences characteristic for crisis periods or intense life situations can have a serious disruptive effect on one's thinking, behavior and actions, and the ability to function in everyday activities or events. Therefore, one of the major challenges of the post-COVID era is supporting mental and physical health by means of specialized strategies and methods.

Reported research as well as practice also shows that, in connection with the psychological consequences of the COVID -19 pandemic, acute depression and post-traumatic stress disorder are currently appearing in people from the medical and helping professions, who worked as the front line workers during the pandemic. There are predictions that COVID-19 will be classified as a diagnosis for many of its consequences in various areas of human health.

A relevant response may be a draft of a specialized online intervention program designed to guide people who are unable to handle the psychological, cognitive and social burden of the pandemic and its consequences.

A major international survey involving 300 respondents from different countries, namely Italy, Spain, Hungary, the UK, the USA, Poland, Slovakia, Romania, Serbia, the Ukraine, and African countries, was conducted from December 2020 until May 2021. Its results may yield meaningful incentives for research and exploration of specific Internet interventions to promote physical and mental health during serious global crises and their aftermath, which influence every aspect of life for each individual. The most severe effects were reported during the COVID19 period as having been the most impactful pandemic event in the modern era.

\section{Methods and results}

The research was carried out from December 2020 until May 2021, during a period when people experienced mental problems as a result of the COVID-19 pandemic, accompanied by depres- sion; long-term stress; chaos; fear; anxiety; frustration; feelings of loss of control over their life or emotions. As a result of such, symptoms of aggression and social phobia; also depression and post-traumatic stress disorder have appeared. The research was carried out on the basis of narrative case studies. To receive an adequate methodological response given the research's focus, which has shown respondents' personal and subjective views, it was necessary to opt for a qualitative approach by using detailed online interviews. Each interview lasted 90 minutes. The aim of the research was to find out how a draft of an online intervention program can help respondents mitigate their problems. The program is people-oriented and focused on stress reduction methods, methods to strengthen mindfulness of the here and now, and some of the relevant theses of Christian missiology and non-radicalized Islam which support human health and dignity.

The precondition for participation in this research was a minimum age of 40 yearsand the experience of at least one consequence of a mental health or long-term psychological burden as a result of the COVID-19 pandemic. Research participants were infected with COVID-19 before the research or they had already recovered from it. Many of them experienced additional health problems; chronic diseases; faced poverty, social exclusion or unprofessional health and social care.

Three hundred respondents took part in the research from various countries: Slovakia, Italy, Spain, Hungary, the UK, Ireland, USA, Poland, Romania, Serbia, the Ukraine, and African countries.

Respondents were contacted via various foreign interest and religious organizations; nongovernmental organizations; self-help groups; international interest organizations focusing on providing support in difficult life situations; psychological support centers; rehabilitation centers; hospitals and healthcare facilities, including those, which have shown a poor level of care.

To ensure the legal protection of the participants and of the research, all respondents provided their consent to GDPR. After initial contact was established via these organizations, groups, centers and hospitals, participants were contacted by the authors of the research via email, various apps or, whenever possible, via the phone. 
As for the African countries, respondents were contacted via community health volunteers and healthcare workers. Contact and participation in the online intervention program was performed in the form of specific consultations with active participation of community health volunteers and healthcare workers. As for respondents from African countries, it was particularly necessary to monitor their perception of demanding and stressful situations and the consequences of the COVID-19 pandemic.

The research team was composed of all the authors of the article. Research participants were contacted and all interviews were organized and a transcript and research notes were written down. The research results were discussed by the authors at a plenary meeting with special attention paid to unusual matters, prejudice and assumptions (e.g. cultural and religious differences). The research captured the respondents' unique experience; however, its limiting factor is the fact that the second part of the research started in May 2021 and, currently, the results cannot yet be applied to the entire world population.

Given the research authors' work and volunteering activities in certain hospitals or healthcare facilities, and given the pandemic situation, face to face contact was limited to a short period of time or completely impossible, and was therefore replaced mainly by phone calls. The research authors have long-standing experience in the area of methods supporting physical health and cognitive functions with an impact on mental health. They demonstrated their professional qualifications in a standard and trustworthy manner to all participants at the very beginning, so that the participants were certain that the contact and the research were reliable. At the first meeting, participants were presented with the research and details about the entire process and ways in which they could participate, and they were informed about their role and the objective and contribution of the research. Participants were also reassured that their anonymity would be guaranteed. At the following first lecture, participants were presented with the benefits of the online intervention program and how it could identify possibilities, which are at the client's disposal, and how it could help them recognize which option was the best for them in a given situation; how it could help the client carefully monitor situations and behavior patterns; which proved to be problematic; and find stimuli allowing them to start the change process. At the lecture, it was also pointed out that the fundamental goal was always the client's autonomy, which should allow them to independently choose among various possibilities and make their own decisions. The lecture explained the importance of the quality of the relationship between the counsellor and the client to make sure that the client feels heard, understood and accepted without prejudice. In addition, the lecture presented several real practical cases which helped the participants understand that the online intervention program could efficiently help clients deal with negative thoughts and emotions and provide them with objective guidance towards personal understanding and growth. Furthermore, the lecture included a presentation of specifics from the area of Christian missiology and non-radicalized Islam which the research authors appropriately adapted to religious differences between research participants. In the case of these specificities, the research authors' intervention is not limited to psychological and social areas; it also focuses on supporting the general inner balance of the client. The aim of these specificities is to promote the healing journey not just for the client but also the environment in which he or she lives.

After the initial lecture, the online intervention program itself was launched with the research participants in the form of 90-minute-long online interviews thrice weekly. The initial interviews within the online intervention program showed that research participants felt like failures because they couldn't handle their situation on their own; felt guilty about needing the help of a counsellor without whom they wouldn't be able to deal with various restrictions related to the pandemic, as well as certain sudden psychological, social and economic consequences of the pandemic which had had a direct impact on their lives. The research participants were also worried that it would be more difficult for them to handle their situation after the online intervention program had finished. In particular, due to negative economic consequences of the COVID-19 pandemic, they experienced great uncertainty and helplessness; many had lost their jobs; and they were afraid to make any plans for the future; as 
a result, apathy could be seen in many of them. The aim of the interventions was not to tell the research participants what they should do or how they should do it, but to encourage them to talk about their worries; to discover the origin of their problems and their way of thinking.

In addition, interventions were based on an encouraging approach of accepting the research participants and an intellectual positive approach based on recognition and respect towards them as human beings (2) with a willingness to see their situation in a more tactful and human approach (2).

After the research participants had identified their biggest worries and uncertainties, they expressed a wish to feel these emotions and worries that had had a significant negative impact on them in a less intense way. At the same time, they wished to start thinking about them in such a manner that they stopped being a mental burden for them.

The research authors then rooted this objective with each of the participants individually based on their needs and, together, they started working on it - first by focusing on positive aspects from each participant's unique point of view, then by redirecting negative thoughts to other positive aspects. Subsequently, the research authors agreed with each participant on the fulfilment of two daily tasks to support the redirection of their negative thoughts into more positive aspects. These tasks were fulfilled by the research participants and assessed together with the research authors every three weeks. After three weeks, the research authors assessed the effect of these tasks with each participant individually and, based on this assessment, they reviewed the objective and modified it to keep it dynamic and motivating for the participant. Then they agreed on further work on this objective with the participant.

The research results can be synthesized into the following areas which should be focused on when providing intervention through specific online counselling even in the post-COVID era. The research participants said that they were trying hard to keep all and even "the smallest feelings of certainty", all positive emotions and the feeling of well-being, which they experienced and gained in online counselling through online interviews with a counsellor. They were worried about "losing these feelings again." Based on these findings, the approach taken by the research authors focused on creating an atmosphere of calm and acceptance during the online interviews in which each participant could think about their following decisions, behavior and actions, and set some important positive goals or activities for themselves; each participant was supposed to start with small goals and activities.

\section{Discussion}

The online intervention program focused on the fact that the research participants could not see an immediate possible solution to their problems. In their interviews with the participants, the research authors tried to identify potential possibilities for each research participant which could be achieved under certain circumstances. The research authors naturally tried to motivate each research participant in their efforts to mobilize their outer and inner resources by redirecting them towards a feeling of hope and by strengthening their feeling of control over their situation. Furthermore, the online intervention program focused on strengthening the research participants' hope and positive thinking and, for this purpose, the research authors agreed with each research participant on the fulfilment of certain special tasks and their subsequent monitoring, completing a general final prognosis of how the participant perceived their problem and the current situation, and how this perception would gradually change with the help of the assigned tasks. The research authors monitored the fulfilment of these tasks in subsequent online interviews every three weeks. After the interviews were over, the research authors assessed the effects of this specially created online intervention program. The aim of certain tasks within this program is to perceive the present moment, to look around oneself and notice what we normally cannot see. Learn to focus on colors, sounds and smells, which the participants had not noticed before. It is essential not to judge, assess or expect anything (Varghese et al. 2020). Based on the assessment, the research authors reviewed the problems with each participant. In the following online interviews, the research participants managed to gradually redirect their perception by focusing on some positive aspects of their situation and then by redirecting their 
thoughts to other positive aspects, which they started to perceive and realize in their interviews with the research authors.

\section{Conclusion}

The international research carried out from December 2020 until May 2021 in Italy, Spain, Hungary, the UK, the USA, Slovakia, Poland, Romania, Serbia, the Ukraine, and African countries, included an online intervention program. Participants were gradually guided towards full autonomy (for example, in thinking and defining their emotions). The program emphasized motivating them to taking responsibility for their own lives and supporting them through a positive assessment of the results achieved in the process. It was proven that it has weakened rumination in the research participants, which was the underlying cause of their mental problems, especially for mood disorders and anxiety disorders, because the research participants focused intensely on the need to activate the process of change. The change process can happen only when stimuli are used to support healthy mental perception, and resulting healthy actions and behaviors replace the unhealthy ones. Moreover, the aim of the online intervention program was to help them find inner balance and a fully-fledged place in society as they used to enjoy before the pandemic. The research results have shown that, in the postCOVID era, special online strategies focused on supporting mental and physical health should not be discarded. Therefore, it is highly relevant for experts engaged in the academic and research fields as well as the practice of online counselling. IT and virtual reality can definitely be enriched with their experience from the COVID-19 pandemic. Professionals should continue taking part in defining, developing and guiding special online methods and strategies to improve quality of life as much as possible. Results show that one of the most important goals is to focus the activity on maximizing the benefits of online special methods and strategies while simultaneously mitigating their risks before, during and after their implementation.

\section{References}

1. TRIZULIAKOVA, J, KRCMERYOVA L, MOJZESOVA, M (2020): Prenatal Palliative Care and Perinatal Hospice - New Chal- lenges in Caring for the Precious Gift of Life in its Fragility. In Clinical Social Work and Health Intervention, 202011 (1) p. 49. ISSN: 2076-9741/online ISSN: 2222-386x/Print DOI 10.22359/cswhi_11_1.

2. VARGHESE, J et al (2020): Certain negative consequences related to the coronavirus pandemic and relevant possible solutions. In Acta Missiologica, 201913 (2) p. 85. ISSN: 1337-7515 (Print) ISSN: 2453-7160 (Online) https://www.actamissiologica.com/. 